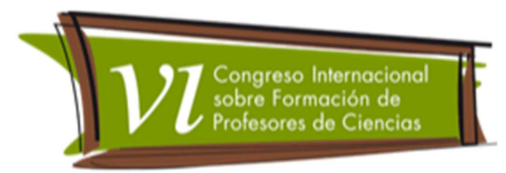

\title{
Caracterización del conocimiento didáctico del contenido curricular de un licenciado en química y de un químico
}

Moreno, William F. '1, Parga Diana L.2

Categoría 2. Trabajo de investigación en proceso.

\section{Resumen}

En Colombia, en los diferentes niveles educativos para la enseñanza de la química, participan dos tipos de docentes, unos que son licenciados en la asignatura y otros que son químicos; sus diversas experiencias son reflejadas en la práctica de aula y demás escenarios educativos. De acuerdo con ello, se ha diseñado una investigación que pretende favorecer el debate sobre las características que posee un docente después de un proceso desarrollado en una universidad formadora de profesores y otra que lo ha preparado para ser un químico y no un profesor en esta área. Por lo tanto, el presente escrito mostrará algunas de las características encontradas respecto al CDC de los dos participantes de la investigación, profesores que enseñan química para carreras de Gestión Ambiental e Ingeniería Ambiental en Universidades de Bogotá.

\section{Palabras Claves}

Conocimiento Didáctico del Contenido, Conocimiento disciplinar, conocimiento histórico-epistemológico, conocimiento psicopedagógico y conocimiento del contexto escolar.

\section{Objetivo}

Caracterizar y analizar el Conocimiento Didáctico del Contenido (CDC) que tiene un licenciado en Química y un Químico, ambos, profesionales dedicados a la docencia de la química en educación superior.

\footnotetext{
1 Candidato a Magíster en Docencia de la Química. Universidad Pedagógica Nacional. elpostigio435@gmail.com

2 Profesora de la Universidad Pedagógica Nacional. dparga@pedagogica.edu.co
} 
Revista Tecné, Episteme y Didaxis: TED. Año 2014, Número Extraordinario. ISSN Impreso: 0121-3814, ISSN web: 2323-0126

Memorias, Sexto Congreso Internacional sobre Formación de Profesores de Ciencias. 08 al 10 de octubre de 2014, Bogotá

\section{El problema}

Dado el debate sobre la pertinencia de los saberes de los licenciados para enseñar y la que tienen otros profesionales cuando enseñan, se ha propuesto indagar: ¿Qué características se identifican en términos del conocimiento didáctico del contenido de los licenciados en química y de los profesores no licenciados cuando enseñan Química? ¿En qué se diferencia su CDC cuando enseñan la Química en la educación superior?

\section{Marco Teórico}

Asumimos que el conocimiento didáctico del contenido es un conocimiento integrador de los conocimientos citados en la figura 1, y que el profesor los organiza de acuerdo con la valoración que le da a cada uno de ellos en su currículo y su práctica profesional. Nos enfocamos en la integración de estos cuatro conocimientos en el currículo construido por el docente determinando qué y cómo enseña química (Mora y Parga, 2008).

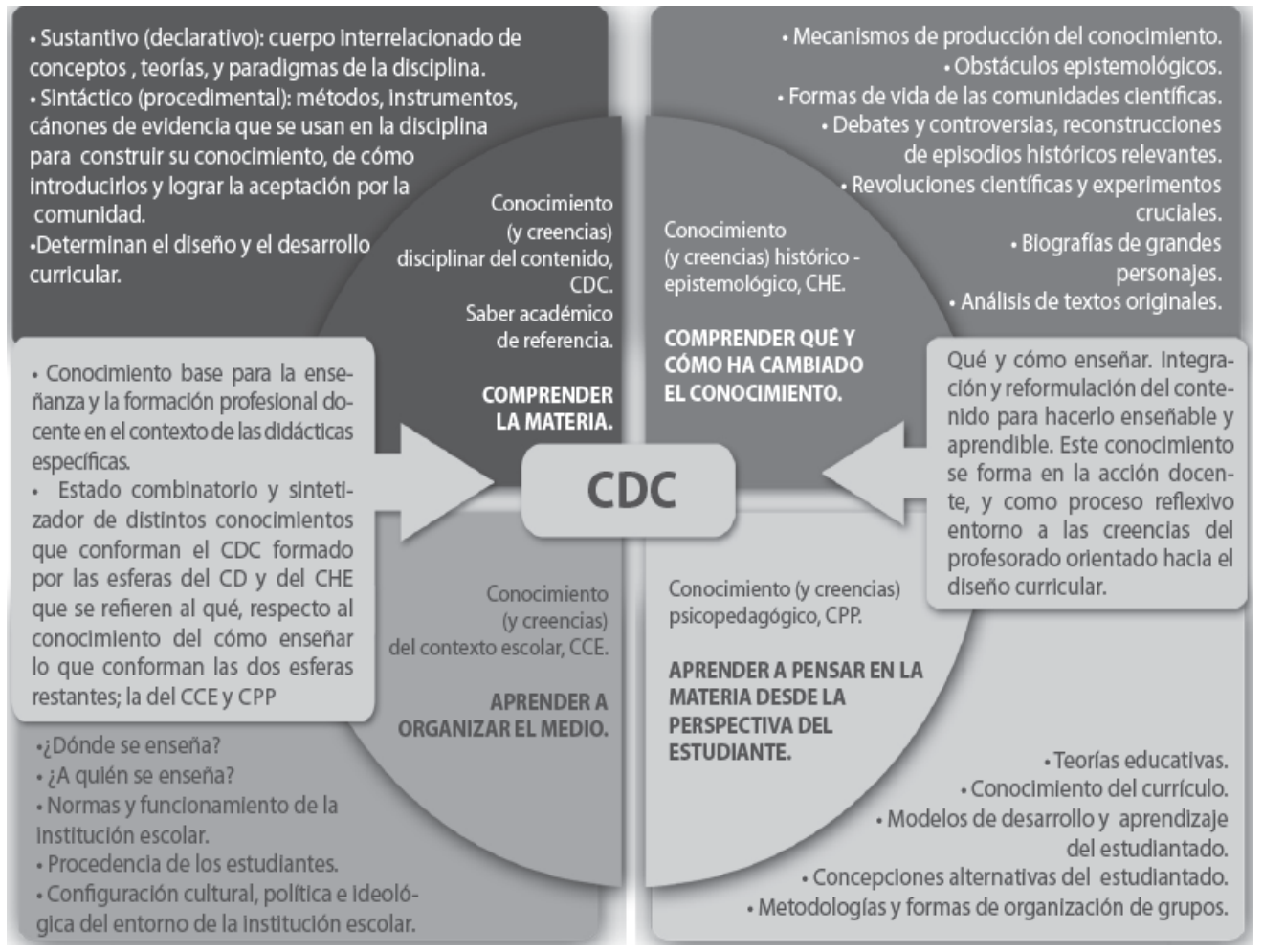

Figura 1. Componentes del CDC. Tomado de Mora y Parga (2008) 
Revista Tecné, Episteme y Didaxis: TED. Año 2014, Número Extraordinario. ISSN Impreso: 0121-3814, ISSN web: 2323-0126 Memorias, Sexto Congreso Internacional sobre Formación de Profesores de Ciencias. 08 al 10 de octubre de 2014, Bogotá

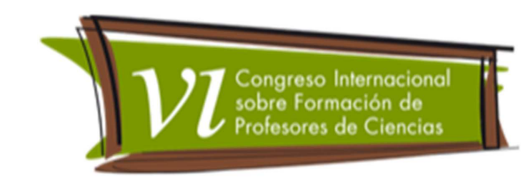

Parga \& Martínez (2007), plantean que hay una diferencia entre el saber específico y el saber didáctico asociado a la enseñanza, en tanto que Parga, Mora \& Martínez (2007) consideran que el profesor necesita transformar los contenidos desde el punto de vista de la comunidad que produce el conocimiento hacia un punto de vista del que necesita enseñarlos, de acuerdo con su intención y su experiencia (Valencia \& Parga, 2009).

\section{Metodología}

El presente trabajo se diseña desde un estudio de caso para caracterizar el CDC mediante las Representaciones de Contenido (ReCo) y el Repertorio de Experiencias profesionales y didácticas (Re-EPD) de los profesores del estudio. Así, el ReCo busca reconocer las grandes ideas a enseñar, los elementos sobre las decisiones curriculares y la reflexión sobre las prácticas docentes. En tanto que el Re-EPD busca reconocer el tipo de evaluación narrativa de su práctica docente, las creencias del profesor acerca de las respuestas de sus estudiantes y lo sucedido en el desarrollo de las clases, el contenido que le da forma a la enseñanza/aprendizaje, y las relaciones que se construyen con los estudiantes (Candela \& Viafara, 2014). Por lo tanto, se han aplicado estos instrumentos, así como el cuestionario del Diseño Curricular (Parga y Martínez, 2007), Grabaciones de Clase, Cuestionarios tipo Likert y Entrevistas (Mulhall, Berry \& Loughran, 2003; Mora \& Parga, 2008). Asimismo el análisis se hace en el marco de las categorías propuestas en el CDC descritas en la figura 1.

\section{Resultados iniciales}

El docente licenciado ha enseñado en la asignatura de química orgánica para la carrera de Ingeniería Ambiental; sin embargo, el químico ha pasado de enseñar química orgánica (Ambiente 1: Ingeniería Ambiental) a química ambiental (Ambiente 2: Gestión Ambiental).

\section{El Caso del Licenciado en Química}

- Frente al Cuerpo interrelacionado de conceptos: las grabaciones y narraciones indican que el profesor práctica en aula una compartimentalización de contenidos, el docente ofrece una explicación del concepto o tema, en este caso la nomenclatura y reactividad en química en orgánica; se evidencia de igual manera que tan pronto explica el tema, se hacen ejercicios o calificación de trabajos, se pasa de una vez a ver el 
Revista Tecné, Episteme y Didaxis: TED. Año 2014, Número Extraordinario. ISSN Impreso: 0121-3814, ISSN web: 2323-0126 Memorias, Sexto Congreso Internacional sobre Formación de Profesores de Ciencias. 08 al 10 de octubre de 2014, Bogotá

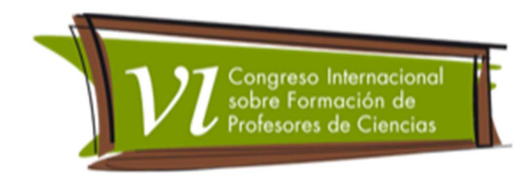

siguiente concepto o tema. El docente cumple lo establecido en los documentos curriculares establecido institucionalmente por los docentes de planta. Aun así en los cuestionarios no se evidencia lo anterior, en sus respuestas pretende enfatizar su enseñanza hacia procesos o destrezas necesarias para que el estudiante afronte su actividad laboral futura.

- Respecto a la categoría Teorías y paradigmas de la química: específicamente se basa en el modelo dual-estructural, explica la nomenclatura y la reactividad de las sustancias orgánicas en términos de sus estructuras moleculares, simplifica la reactividad de las sustancias mediante algoritmos consistentes en identificar los grupos funcionales de los reactantes y de acuerdo con estos, la obtención de productos con diferentes grupos funcionales.

- Sobre las Teorías educativas: es evidente su afinidad por el modelo de transmisión-recepción combinada con la demostración en laboratorio, le interesa entonces que los estudiantes vean cómo por medio de procedimientos pueden llegar a solucionar problemas afines a su actividad laboral, en ello explica las ideas y conceptos que subyacen como guías necesarias para recordar y orientarse en la solución de problemas. Las evaluaciones consisten en una calificación de preguntas abiertas que evidencie la utilización de estos procesos o procedimientos en sus respuestas.

- Sobre el Conocimiento del currículo: en su proceder en el aula evidencia la idea de una lista de conceptos, destrezas y procesos que deben superarse a mediad que hace una hetero-evaluación. $Y$ en las respuestas de los cuestionarios afirma que no necesita una planificación curricular pues ya establecida por la institución.

- Concepciones alternativas: no las caracteriza ni hace una reflexión profunda de estos en los estudiantes.

- Metodologías en las formas de organización de grupos: el docente como tal organiza su clase en momentos caracterizados en actividades, las cuales consisten: en explicación, cuyos estudiantes se ordenan al frente del tablero y hacen sus anotaciones; resolución de talleres y/o trabajos que se organizan predominantemente en grupos con asesoría continua de él o de compañeros experimentados; y finalmente, evaluación, los estudiantes responden individualmente sin posibilidad de apoyarse en otros compañeros o en el profesor.

- Debates y contribuciones históricas: el docente explica anecdóticamente eventos históricos de la química, pero no la profundiza ni la evalúa, aunque 
estas referencias históricas podrían dinamizar la explicación enseñando historia y epistemología.

- En los cuestionarios el docente expresa la importancia de este ítem, enfocado simplemente a profundizar el conocimiento disciplinar y no otros tipos de conocimientos.

- Características de los estudiantes: son estudiantes que trabajan durante el día y en la noche estudian. Esto ha influido en la poca dedicación extra-clase para repasar y solucionar tareas.

- Ampliación del currículo por medio del uso de las tecnologías de información y comunicación: no se dedica a diseñar apoyos en este aspecto y no lo amplía su currículo; basta con lo determinado por la institución y su equipo de planta.

- Proceso Formativo del profesor: su gusto por la química partió de la iniciativa propia de hacer las prácticas de laboratorio que otros grupos realizaban cuando él colaboraba como laboratorista (en marco de un programa universitario que apoyaba a estudiantes con restricciones económicas). Es evidente su postura frente a la química pero no hacia la enseñanza de esta, narra su experiencia de laboratorio en asignaturas como química general y química analítica, orgánica e inorgánica; menciona que en las primeras se siguen recetas de "cocina" y en las restantes se planteaban propuestas en torno a identificación de sustancias.

- Asignatura Química Orgánica: el docente fue observado en dos semestres diferentes, factor que no parece incidir en la caracterización de su CDCC.

\section{El Caso del químico}

- Sobre el Cuerpo interrelacionado de conceptos: el profesor maneja una visión jerarquizada y compartimentalizada; en la práctica de aula él impone un criterio para seleccionar los temas y/o conceptos más importantes, organiza según esta selección los temas que explica a lo largo del tiempo; sin embargo, explica las características más importante de un tema y acto seguido comienza a explicar otro ítem diferente; este profesor es precavido de que los temas tengan un hilo conductor coherente y que sus prácticas respondan a estos.

El profesor en el ambiente 1 intenta solo enfatizarse en el reconocimiento de sustancias químicas orgánicas con sus respectivos grupos funcionales pero no trata a profundidad la reactividad, exceptuando en el tema de ácidos donde considero importante desarrollar la síntesis de esteres por medio de la esterificación de Fisher. 
Revista Tecné, Episteme y Didaxis: TED. Año 2014, Número Extraordinario. ISSN Impreso: 0121-3814, ISSN web: 2323-0126 Memorias, Sexto Congreso Internacional sobre Formación de Profesores de Ciencias. 08 al 10 de octubre de 2014, Bogotá

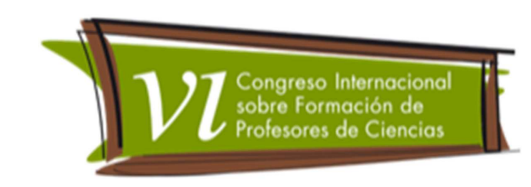

- En las Teorías y paradigmas de la química: en el Ambiente 1 el profesor utiliza la teoría dual-estructural para explicar nomenclatura y reconocimiento de grupos funcionales; en el Ambiente 2 el profesor utiliza la teoría precuánticocuántico, siendo curioso la forma de construir un híbrido entre los dos, para explicar la configuración electrónica, explico la periodicidad de los elementos relacionando la configuración electrónica con sus comportamiento químico; en sus prácticas de laboratorio fue demostrativas.

- Frente a las Teorías educativas: se apoya en el modelo de transmisiónrecepción, combinado con la demostración característica de laboratorio; el profesor es bien hábil en captar la atención de los estudiantes por medio de la jocosidad y desarrolla la explicación ganándose la confianza de los estudiantes lo que facilita que los estudiantes pregunten elementos que no comprenden.

- En el Conocimiento del currículo: lo concibe como el listado de conceptos, procesos y destrezas, aun así se preocupa por ofrecer elementos en los cuales los criterios de los estudiantes se amplié en el momento de la toma de decisiones, en este sentido su enseñanza se puede traducir en dividir su currículo en las causas y consecuencias de ciertas problemáticas profesionales acordes a su futura actividad laboral, aunque su práctica en aula no deja en claro esta intención.

- Concepciones alternativas: explícitamente no muestra una postura sobre el ítem, pero en el aula el docente pregunta y explica situaciones jocosas para que el estudiante evidencie estas concepciones pero su forma de concebirlas son como errores susceptibles de ser corregibles.

- Metodologías en las formas de organización de grupos: la organización del grupo es completamente magistral, con experimentos demostrativos.

- Debates y contribuciones históricas: en su práctica de aula no evidencia utilizar la historia ni la epistemología para la enseñanza; pero en los cuestionarios indica que utiliza la historia y epistemología para demostrar la evolución del concepto.

- Características de los estudiantes: en el Ambiente 1, los estudiantes trabajan de día y en la noche estudian; en el Ambiente 2, los estudiantes no trabajan y dedican una gran parte de su tiempo y recursos al estudio.

- Ampliación del currículo por medio del uso de las tecnologías de información y comunicación: menciona la intencionalidad de utilizarlos, aún así n evidencia en su práctica ningún criterio para la ampliación de su currículo con estos.

- Proceso Formativo del profesor: su gusto por la química partió de la enseñanza en el colegio de un profesor quién era ingeniero químico y químico, este 
Revista Tecné, Episteme y Didaxis: TED. Año 2014, Número Extraordinario. ISSN Impreso: 0121-3814, ISSN web: 2323-0126

Memorias, Sexto Congreso Internacional sobre Formación de Profesores de Ciencias. 08 al 10 de octubre de 2014, Bogotá

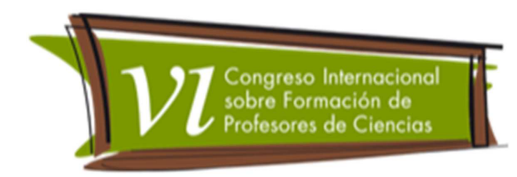

generaba un ambiente de confianza con rutinas de chistes durante 10 minutos; le orientó en escoger la carrera e influyó en sus creencias y estilo de enseñanza.

- Asignatura Química Orgánica y Química Ambiental: el docente fue observado durante dos semestres diferentes, factor que no parece incidir en la caracterización de su CDCC.

\section{Conclusiones}

Los primeros resultados permiten encontrar los siguientes aspectos comunes en el CDC de los dos profesores:

- Dan un peso predominante al conocimiento disciplinar, su enseñanza depende de la transposición didáctica del conocimiento y no una integración, en este caso, usan analogías o ejemplos para relacionar lo enseñado con el conocimiento cotidiano de los estudiantes.

- El interés para ambos docentes se centran en dar herramientas ya sea de interpretación y ampliación de criterios para su futura actividad laboral.

- La evaluación se centra en corregir las formas incorrectas de respuestas, y ambos convergen en entenderlos como los exámenes escritos con preguntas abiertas y trabajos.

- La actividad en el laboratorio se centra en demostrar lo que han visto en el aula y atender dudas esporádicas de los estudiantes.

- Tienen preocupación por el tiempo en los encuentros con los estudiantes y tienden a considerarlo importante para la enseñanza de la química limitando su práctica docente. No amplían su currículo con la TIC.

- Ambos docentes no tienen en cuenta su enseñanza de acuerdo con el contenido disciplinar de la asignatura.

- Parece ser que la asignatura no influye en los criterios de enseñanza que adoptan.

Respecto a las diferencias encontradas se resaltan:

- Para el licenciado el conocimiento previo no parece ser de importancia, mientras que para el químico es importante la adquisición de definiciones previas asociadas al contenido.

- La enseñabilidad es entendida por el licenciado en términos del contenido disciplinar; el uso de la historia es anecdótico; para el químico no se entiende el concepto y por lo tanto no se atreve a responder; sin embargo, en lo 
Revista Tecné, Episteme y Didaxis: TED. Año 2014, Número Extraordinario. ISSN Impreso: 0121-3814, ISSN web: 2323-0126

Memorias, Sexto Congreso Internacional sobre Formación de Profesores de Ciencias. 08 al 10 de octubre de 2014, Bogotá

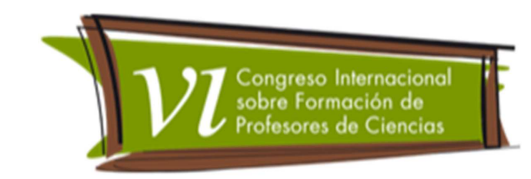

referente a la historia lo utiliza para apoyarse en explicar la evolución histórica del concepto.

- Para el docente químico el diseño curricular se sujeta completamente a los documentos oficiales de la institución, dedicándose a exponer el contenido; sin embargo, el licenciado diseña talleres y guías de laboratorio.

- Su formación docente no determina su estilo de enseñanza, sino la forma en que le enseñaron química; el Licenciado en Química se enfoca en explicaciones teóricas y prácticas demostrativas, mientras que el Químico, con esta misma tendencia, crea un ambiente de confianza por medio de la jocosidad dentro del aula y el laboratorio.

\section{Referencias bibliográficas}

Albion, P., Finger, G., \& Jamenson-Proctor, R. (2010). Beyond pedagogical content knowledge: the importance of TPACK for informing preservice teacher education in australia. Inernational federation for information processing(AICT 324), 114-125.

Candela, B. F., \& Viafara, R. (2014). La implimentacion de los ReCo y los PaP-eRs en la formación de docentes. Tecné, Epsiteme y Didaxis: TED.

Hernadez, R., Fernández, C., \& Baptísta, P. (2006). Metodología de la investigación. México: McGraw-Hill.

Mora, W. \& Parga, D. (2007). Tramas histórico-epistemológicas en la evolución de la teoría estructural en química orgánica. Tecné, episteme y didaxis(21).

Mora, W., \& Parga, D. (2008). El conocimiento didáctico del contenido en química: integración de las tramas histórico-epistemológicas con las tramas de contexto-aprendizaje. Tecné. Episteme y Didaxis, 56-81.

Mulhall, P., Berry, A., \& Lougrhan, J. (2003). Frameworks for representing science teachers'pedagogical content knowledge. Asian-Pacific forum science learning and teching.

Perilla, J., \& Parga, D. (2009). Conocimiento didáctico del contenido curricular, el diseño en la formación permanente de profesores de química. Tecné, Episteme y Didaxis(Extraordinario). 
Revista Tecné, Episteme y Didaxis: TED. Año 2014, Número Extraordinario. ISSN Impreso: 0121-3814, ISSN web: 2323-0126

Memorias, Sexto Congreso Internacional sobre Formación de Profesores de Ciencias. 08 al 10 de octubre de 2014, Bogotá

Posner, G. (2004). Análisis del currículo. Mc Graw Hill.

Valencia, F., \& Parga, D. (2009). Caracterización del conocimiento didáctico del contenido curricular en los profesores de ciencias al diseñar una unidad didáctica con enfoque ciencia, tecnología, sociedad y ambiente (CTSA). Tecné, Episteme y Didaxis(Extraordianrio). 